\title{
A Research on Use of Robotics In High Rise Buildings
}

\author{
Vinod M Kapse, Aditee Mattoo
}

\begin{abstract}
A Growing Number of High-Rise structures have been all being Launched in populated countries such as China together using living standards' requirement. But around the 1 hand, with all the evolution of an aging culture, the more labor deficit is getting an issue; over the flip side, the threat and also difficulties at building to increase with all the elevation of these properties. Ergo, automation and robotics technology's applying is forecast to facilitate considerations and troubles. As far as now, approaches and many different procedures with robotics and automation engineering are suggesting to function as utilized in construction structure. This paper implies instructions of research also outlines the progress on this use of robotics and automation technologies in construction structure. To start with, the study range, the procedure and also the literature survey's outcome have been all presented. Afterward, dependent on the poll outcome, the most relevant software are outlined in regards to the 3 components of high tech structures, i.e., ground and base operate, superstructure erection along with façade setup. At length, chances and the instructions cited in current research have been outlined and reviewed. This paper gives an invaluable asset of robotics and automation engineering for structures.
\end{abstract}

Keywords - Robotics, automation, high rise buildings.

\section{INTRODUCTION}

As a result of financial and market progress, this world buildings' variety is slowly growing. The development of properties mitigates the anxiety about their population but in addition take a huge number of labor force. The number of personnel is diminishing as a result of aging people in most nations on the planet, in the building site. Moreover, the basic security issues and also the employees' panic of this elevation would be additionally struggling in high tech buildings' building site. Labor deficit and also the lifetime security of these workers involve Implementing robots and devices to restore human labor.The applying of robotics and automation technologies within the building industry are examined in lots of newspapers. Bock [1] implied that construction strategy experienced achieved its limits and summarized five different areas of chances for building automation style and layout industrialization, structure robots, robots, internet web site automation, and even robotics. Son et al.examined the contents and topics of 1671 newspapers in International Symposium on Robotics and Automation in Construction (ISARC) proceeding from 1990 into 2008, to recognize developments and prospective instructions of automation and robotics engineering in

Revised Version Manuscript Received on 10 September, 2019.

Dr. Vinod M Kapse, Electronics and Communication Engineering, Noida Institute of Engineering and Technology, Noida, U.P. India.

(Email: research.paper@niet.co.in)

AditeeMattoo, Information Technology, Noida Institute of Engineering and Technology, Noida, U.P. India.

(Email: research.paper@niet.co.in) structure. Vähäet al. [3] researched on detector engineering and its prospective usage for development automation and also examined autonomous software at the principal structure processes. Taylor et al. [4] outlined the prevailing automatic machines and structure robots in Japan and implied construction surroundings need to become more ordered and commanded to employ robots. Maas and van Gassel [5] reviewed the evolution of robotics and automation technologies along with its own influence on efficiency direction, structure technology, and development administration. These newspapers directed from that exactly the essential research fields have introduced the usage of robotics and automation technological innovation within the building sector as an entire and examined the analysis and growth tendencies. But, is a shortage of some methodical inspection of this usage of robotics and automation engineering in construction structure. This paper outlines the progress over the use of automation and robotics suggests instructions of research also technologies by means of a literature study in construction structure. For this end the paper is targeted on several sub-phases of construction structure: superstructure erection, ground and base to operate along with structure. In Part two, the procedure, the study range and also the outcome of the literature questionnaire have were all presented. By assessing the poll outcome, Sections 3, 5 and 4 present the applying of automation and robotics technologies inside the building of the superstructure base along with façade, and Section 6 outlines the instructions of robotics and automation technologies for structures.

\section{LITERATURE REVIEW}

This is of this definition of "highrise construction" changes in various nations. By way of instance, based on Chinese specifications, a high tech construction means residential structures within ten testimonies or $28 \mathrm{~m}$, or even nonresidential structures within $24 \mathrm{~m}$; at Japan, a high-tech construction is eight testimonies or $31 \mathrm{~m}$; and also at the U.S.," it's finished 24.6 four or m legends. In any case, the highrise properties taller compared to one hundred $\mathrm{m}$ have been named "Sky Scrapers". The higher - growth construction isn't rigorously characterized plus the definitions are covered by additionally and comprises the Sky Scrapers.To execute a literature review, an all-inclusive investigation was run for example net of Science, Scopus," ASCE, and Engineering Village. The hunt key words 
comprised"highech","robot","automation","structure","asse mbly","setup","equipment","gear", etc.. The books within the shape of editorials, novels, conversations, or printed have been excluded as they're regarded as of significance to its inspection. We pumped our newspapers last but not least got 50 newspapers After looking at the names, abstracts and decisions. Specially we located the inspection on the application of automation and robotics engineering at the sub-phase of ground and also base work have already been all-inclusive (that's farther clarified in part 3), thus we just comprised about several latest inspection newspapers of ground and also base job inside this review.

\section{APPLICATIONS}

The ground and base function are a sub stage par phase for every single kind of buildings' development. This can be among the very damaging job in construction structure and comprises surgeries. The study to this use of robotics and automation technologies in the base and the world job is just one of those leaders within the building business, and also newspapers are published with this subject. Reviews are done to now outline the most studies. This analysis gift ideas several reviews to steer clear of perform.Naskoudakis et al. [6] reviewed 73 newspapers posted from 2006 to 2015 about earthmoving gear and split into seven major research topics, 1 which will be robotics and automation. 2 sub-themes had been subsequently recognized, i.e., un-manned structure, along with also real- time detection and monitoring. Kamat and azar [7] gave an in depth report on their progress in automation in the academic and industry network and also discussed prospective instructions and that the limits. One of the previous individual has been defined since the disadvantaged fields they categorized the research of tools automation to four different groups: tools monitoring and fleet management, protection administration, products present estimation and equipment controller, handheld remote management and performance. Dadhich et al. [8] given the foundation of this autonomous earthmoving and also presented with a questionnaire since the essential search themes and recognized that the comprehension openings of automation of earthmoving devices, notably the performance of wheel loaders at a brief loading cycle. Inside this assessment paper, the information gaps are recognized as communicating for performance, excavation, and the operator practical encounter throughout performance.

For its sub-phase of all both superstructure erection of high- rise properties, the building issue rises considerably since the construction stature develops, which chiefly arises in several elements, both the very minimal protection and productivity factors as a result of manual perform from toxic working setting, the perpendicular transport of staff, equipment and materials, and also gathering of significant items, notably to steel constructions. The research that are relevant are grouped to about a few for the 3 aspects: automatic mounting processes, automated structure devices, along with also metal construction procedures. They will be described by the:

\section{AUTOMATED CONSTRUCTION SYSTEMS}

Structure procedures have been made to stabilize the structure procedure by setting an operating environment that's acceptable to employ robots and the equipment and enhance the growth of construction structure. 7 papers that were connected were also identified. Miyakawa et al. [9] suggested an automatic construction structure technique (ABCS) for high speed steel constructions, that incorporates a few pieces: 1)) a synchronously scaling structure which enclose an off-beat working distance, termed "tremendous Construction Mill (SCF)"; two) a parallel shipping platform to get substance lifting; and even also 3) concentrated direction approaches, for creation direction, device's functionality direction and equipment controller. Predicated on ABCS, the other approach known as "huge Canopy" has been formulated for pre-fabricated highrise concrete properties [10, 11], that adheres for the traits of concrete structure.In comparison to the traditional way, the functioning terms and structure growth enhanced. Nevertheless, this SCF's apparatus arrangement restricts the versatility of construction methods that are automated. Furthermore, weights and the costs are high on account of these robots' mixture cranes and lifting processes [12, 13]. Hence, Kang et al. [1-2] established a robotic-crane established automatic structure technique (r-a platform) for metal constructions of high tech structures, that contains a structure mill (CF), also a steel gathering platform and also a tracking and management platform. Back in a ca program, the tower was put in at the heart of construction the major robot and lifting technologies have been somewhat separate of their $\mathrm{CF}$, that reduced the burden of this system and also lower the overall structure cost in addition to providing a suitable distance. Kim et al. [1 3] designed a few Profession $\mathrm{CF}$ solutions to enhance the operating environment and assessed them with all the Computational Fluid Dynamics strategy. Results and also the test procedure given a way to choose the $\mathrm{CF}$ in construction structure. With the structure system's evolution, appraisal processes were suggested to assess construction efficacy and the economic. Kim et al. [14] established a benefit/cost identification course of action for its applying form of robot-based structure automation approaches, that may quantify the financial viability of their automated techniques. Van Gassel [1-5] ran a simulation of the higher - rise building's development means, including using a structure strategy. By assessing also the construction information and the outcome of the newspaper encouraged the usage of construction procedures at holland, also affirmed that the advantages of construction procedures.

\section{AUTOMATED LIFTING SYSTEM\& RESULTS}

For structures, the structure efficacy is greatly influenced by the transport of equipment, materials and staff. Various scientific reports are executed to boost the overall automation amount of their transport devices. Eight papers that were related are all identified.Kim et al. [16] suggested a desk form-work lifting platform incorporated with structure hoists, which improve the productiveness, cuts the substantial preliminary price and warrants extra job with meeting and performance. With this particular specific tool kit an 
elevator car might be transformed into an individual, that may make use of communications technology and remote detection take care of it and to catch substance movement's advice. Predicated with this tool kit, an idea of the elevator that is good is suggested, together using broadcasts of both wireless communications and functionality.Optimization and operation planning are all aspects to fix the efficacy of this lifting procedure that is automated. Sin et al. [19, 20] suggested an unmanned clever lifting platform also invented an optimized performance algorithm for either double or multi-cage lifts, and that may decrease work hours and targeted visitors jams. Cho et al. [2-1] given a simulation procedure for development hoists to figure out the lifting cycle period based to lifting loads and heights, and also to build a perfect functioning prepare. Additionally they acquired an algorithm [2-2] in structures for course preparation of their performance that was multi-lifting. All these calculations and processes optimized for the charge of the functioning apparatus, and enriches the operation efficacy of shipping.Operation tracking another major element, particularly for your tower cranes that are wise. Lee et al. [2-3] suggested an automatic lifting-path monitoring technique to get a robotic tower crane, that utilized laser apparatus to assess the terminal space, along with also an encoder and also an accelerometer to assess the vertical and horizontal angles. The procedure can be effective in raising course preparation and safety direction and tracks the performance activities of this tower.

\section{CONCLUSION}

This paper outlined the usage of automation and robotics engineering in high tech construction structure from fifty newspapers posted from 2000 to 2017 to supply a systematic report on this modern analysis and improvements within this discipline by the view of 3 big structure sub-phases on the ground and also base operate, superstructure erection dysfunction, along with façade setup. The newspaper additionally spoke future instructions in every single subperiod at structure dependent around the literature. For the reason which offers a very important aid of automation and robotics technologies for 29 , this newspaper is predicted to add.

\section{REFERENCES}

1. K. S. Rabe, J. Mueller, M. Skoupi, and C. M. Niemeyer, "Cascades in Compartments: En Route to Machine-Assisted Biotechnology," Angew. CHEMIE-INTERNATIONAL Ed., vol. 56, no. 44, pp. 13574-13589, Oct. 2017.

2. H.-J. Su, C. E. Castro, A. E. Marras, and L. Zhou, "The Kinematic Principle for Designing Deoxyribose Nucleic Acid Origami Mechanisms: Challenges and Opportunities,” J. Mech. Des., vol. 139, no. 6, Jun. 2017.

3. J. Wajcman, "Automation: is it really different this time?," Br. J. Sociol., vol. 68, no. 1, pp. 119-127, Mar. 2017.

4. M. Li, L. Liu, N. Xi, and Y. Wang, "Applications of Micro/Nano Automation Technology in Detecting Cancer Cells for Personalized Medicine," IEEE Trans. Nanotechnol., vol. 16, no. 2, pp. 217-229, Mar. 2017.

5. L. Yang, R. Said, and M. Abdel-Rehim, "Sorbent, device, matrix and application in microextraction by packed sorbent (MEPS): A review," J. Chromatogr.
B-ANALYTICAL Technol. Biomed. LIFE Sci., vol. 1043, pp. 33-43, Feb. 2017.

6. G.-Y. Gu, J. Zhu, L.-M. Zhu, and X. Zhu, "A survey on dielectric elastomer actuators for soft robots," Bioinspir. Biomim., vol. 12, no. 1, Feb. 2017.

7. P. Rytlewski, B. Jagodzinski, and K. Moraczewski, "Laser-Assisted Electroless Metallization of Polymer Materials," in PROGRESS IN ADHESION AND ADHESIVES, VOL 2, Mittal, KL, Ed. 2017, pp. 321-350.

8. R. Dowsett, C. Harty, and R. Davies, "The complete robot? The human-machine interface in temporary off-site construction work," in PROCEEDINGS OF THE 9TH NORDIC CONFERENCE ON CONSTRUCTION ECONOMICS AND ORGANIZATION, 2017, pp. 93-103.

9. H. Ivan Tangarife and A. Escobar Diaz, "Robotic Applications in the automation of agricultural production under greenhouse: a review," in 2017 IEEE 3RD COLOMBIAN CONFERENCE ON AUTOMATIC CONTROL (CCAC), 2017.

10. C. Wang, L. Ikuma, J. Hondzinski, and M. de Queiroz, "Application of Assistive Wearable Robotics to Alleviate Construction Workforce Shortage: Challenges and Opportunities," in COMPUTING IN CIVIL ENGINEERING 2017: SENSING, SIMULATION, AND VISUALIZATION, 2017, pp. 358-365.

11. J. Muhammad, H. Altun, and E. Abo-Serie, "Welding seam profiling techniques based on active vision sensing for intelligent robotic welding," Int. J. Adv. Manuf. Technol., vol. 88, no. 1-4, pp. 127-145, Jan. 2017.

12. M. W. Bowler, O. Svensson, and D. Nurizzo, "Fully automatic macromolecular crystallography: the impact of MASSIF-1 on the optimum acquisition and quality of data," Crystallogr. Rev., vol. 22, no. 4, pp. 233-249, Oct. 2016.

13. A. Bechar and C. Vigneault, "Agricultural robots for field operations: Concepts and components," Biosyst. Eng., vol. 149, pp. 94-111, Sep. 2016.

14. D. I. Onwude, R. Abdulstter, C. Gomes, and N. Hashim, "Mechanisation of large-scale agricultural fields in developing countries-a review," J. Sci. Food Agric., vol. 96, no. 12, pp. 3969-3976, Sep. 2016.

15. J. Iqbal, R. Ul Islam, S. Z. Abbas, A. A. Khan, and S. A Ajwad, "AUTOMATING INDUSTRIAL TASKS THROUGH MECHATRONIC SYSTEMS - A REVIEW OF ROBOTICS IN INDUSTRIAL PERSPECTIVE," Teh. Vjesn. Gaz., vol. 23, no. 3, pp. 917-924, Jun. 2016.

16. M. Vazquez-Arellano, H. W. Griepentrog, D. Reiser, and D. S. Paraforos, "3-D Imaging Systems for Agricultural Applications-A Review," SENSORS, vol. 16, no. 5, May 2016 